\title{
醋酸银/甲醇促进炔基膦酸酯水合制备 $\beta$-羰基膦酸酯
}

\author{
刘开建* 欧金花欧丽娟刘宏伟 \\ 唐新德李来丙胡波年 \\ (湖南工学院材料与化学工程系 衡阳 421002)
}

\begin{abstract}
摘要 报道了一种高原子经济性、高选择性合成 $\beta$-羰基膦酸酯类化合物的催化水合体系. 实验发现以廉价的 $\mathrm{AgOAc}$ 作为催化剂, 甲醇作为助催化剂, 炔基膦酸酯顺利发生水合反应生成相应的目标产物, 产率优秀. 反应无需惰性气体 保护，具有良好的官能团耐受性，杂环、卤素等官能团及杂环体系都对反应无明显影响.
\end{abstract}

关键词 炔基膦酸酯; $\beta$-羰基膦酸酯化合物; 银催化; 水合反应

\section{Effective Transformation of Alkynylphosphonates into $\beta$-Ketophosphonates with a Silver(I) Catalyst}

\author{
Liu, Kaijian* Ou, Jinhua $\quad$ Ou, Lijuan Liu, Hongwei $\quad$ Tang, Xinde \\ Li, Laibing $\mathrm{Hu}$, Bonian \\ (Department of Materials and Chemical Engineering, Hunan Institute of Technology, Hengyang 421002)
}

\begin{abstract}
Ketophosphonates are an important class of carbonyl compounds which exhibit a wide range of biological activities and outstanding metal-complexing abilities. They are building blocks in organic synthesis, especially as intermediates for the synthesis of $\alpha, \beta$-unsaturated carbonyl compounds, chiral $\beta$-hydroxy and $\beta$-hydroxy phosphonic acids. In this paper, a cheap, simple, convenient and environmentally benign method to synthesize the $\beta$-ketophosphonates from alkynylphosphonates was developed, which can be easily prepared in one step from commercial terminal alkynes. In the presence of $10 \mathrm{~mol} \% \mathrm{AgOAc}$ and 4 equiv. of water in methanol at $110{ }^{\circ} \mathrm{C}$, a broad range of alkynylphosphonates were converted into the corresponding $\beta$-ketophosphonates in nearly equivalent conversion through silver/methanol-catalyzed hydration reaction. The presented methodology will provide new strategies for $\beta$-ketophosphonates-type natural product and drug synthesis, which has important academic significance and application value.
\end{abstract}

Keywords alkynylphosphonates; $\beta$-ketophosphonate; silver catalysis; hydration reaction

$\beta$-羰基膦酸酯是一种非常重要的有机化合物, 被广 泛应用于有机合成 ${ }^{[1 \sim 4]}$ 、药物化学 ${ }^{[5 \sim 9]}$ 等多种领域中. 例 如, $\beta$-羰基膦酸酯化合物常被用于 $\alpha, \beta$-不饱和羰基化合

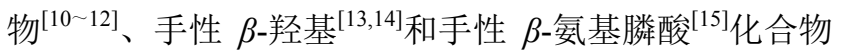
的合成中. 同时, $\beta$-羰基膦酸酯作为一类重要的配体, 在金属催化化学及配位化学中也发挥着重要的作 用 ${ }^{[16,17]}$. 由于它的重要性和应用性, 受到有机化学工作 者的重视. $\beta$-羰基膦酸酯类化合物的传统合成方法包括 Arbuzov 反应、烷基膦酸酯酰基化反应等 ${ }^{[18-20]}$. 炔烃的
水合反应是合成羰基化合物的重要方法，具有原子经济 高、环境友好等特点 ${ }^{[21 ~ 24]} .1966$ 年, Sturtz 等 ${ }^{[25]}$ 首次报道 录盐高效催化炔基膦酸酯水合反应制备 $\beta$-羰基膦酸酯 化合物. 2012 年, 赵玉芬课题组 ${ }^{[26]}$ 报道了氯化钯催化的 炔基膦酸酯水合反应. 最近, 我们课题组 ${ }^{[26,27]}$ 报道了阳 离子金催化剂催化卤代炔烃及炔基膦酸酯水合反应. 然 而，在这些方法中还存在一些不足，如使用一些有毒或 者价格昂贵的金属催化剂. 因此, 鉴于我们已有的在炔 基水合反应方面的工作基础 ${ }^{[27,28]}$ ，希望构建另外一种新

*E-mail: liukaijian_1982@163.com; hbonian@163.com

Received February 8, 2015; revised May 1, 2015; published online July 10, 2015.

Project supported by the Science and Technology Planning Project of Hunan Province (No. 2014FJ3053), the National Natural Science Foundation of China (No. 21176061), the Natural Science Foundation of Hunan Province (No. 13JJ3132), the Construct Program of the Key Discipline in Hunan Province and Science and Technology Planning Project of Hengyang City (No. 2014KJ26).

湖南省科技计划(No. 2014FJ3053)、国家自然科学基金(No. 21176061)、湖南省自然科学基金(No. 13JJ3132)、湖南省重点建设学科和衡阳市科技计划 (No. 2014KJ26)资助项目. 
的方法, 用廉价且环境友好的银作为催化剂来实现炔基 膦酸酯的水合反应, 为合成 $\beta$-羰基膦酸酯提供一条简单 有效的新的合成路径.

\section{1 结果与讨论}

\section{1 反应条件的优化}

以 2-葵基膦酸二乙酯(1a) $(0.3 \mathrm{mmol}) 、 10 \mathrm{~mol} \%$ $\mathrm{AgSbF}_{6}$ 、甲醇 $3 \mathrm{~mL}$ 、水 $1.2 \mathrm{mmol}$ 为投料比, 用薄层色 谱(TLC)监控反应, 室温下反应 $24 \mathrm{~h}$ 后发现, 扔有大量 原料末反应, 气相色谱分析证明底物 $1 \mathrm{a}$ 的转化率仅 13\%, 相应水合产物(2-葵基-2-氧代)膦酸二异乙酯(2a) 收率为 11\% (Table 1, Entry 1). 用上述投料比, 2-葵基膦 酸二乙酯为模板底物, 进行反应条件优化, 实验结果见 Table 1.
首先筛选了反应温度，结果表明，随温度的升高， 底物转化率也随之提高, 当温度达到 $110{ }^{\circ} \mathrm{C}$ 后转化率 没有显著的变化(Table 1, Entries 2 6). 接着篮选了不 同银盐(Table 1, Entries 7 13), 发现 AgOAc 是该反应 的最佳催化剂(Table 1, Entry 10, 分离收率 96\%). 然后 考察了催化剂装载量对反应的影响, 发现增加装载量至 $15 \mathrm{~mol} \%$, 底物的转化率没有明显的改变; 然而降低催 化剂的装载量至 $5 \mathrm{~mol} \%$, 反应的转化率大幅降低(Table 1, Entries 14, 15). 在以纯水为溶剂时产率低下(Table 1, Entry 16), 这可能是因为在纯水中产物溶解度较低的缘 故. 最后, 我们对溶剂进行了篮选(Table 1, Entries 16 $20)$, 结果证实甲醇依然是最优选择. 研究还发现铁盐、 酮盐及质子酸都不适合催化炔基膦酸酯的水合反应 (Table 1, Entries 21 24). 最终我们确定了最佳反应条

表 1 反应条件优化 ${ }^{a}$

Table 1 Optimization of reaction conditions

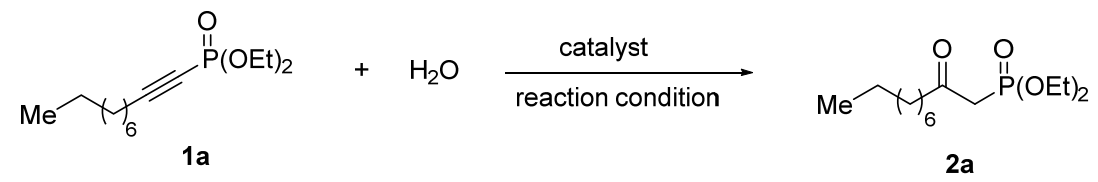

\begin{tabular}{|c|c|c|c|}
\hline Entry & Catalyst & Reaction condition & Conversion $c / \%$ \\
\hline 1 & $10 \mathrm{~mol}^{2} \mathrm{AgSbF}_{6}$ & $\mathrm{CH}_{3} \mathrm{OH}$, r.t. & 13 \\
\hline 2 & $10 \mathrm{~mol}^{\%} \mathrm{AgSbF}_{6}$ & $\mathrm{CH}_{3} \mathrm{OH}, 40{ }^{\circ} \mathrm{C}$ & 31 \\
\hline 3 & $10 \mathrm{~mol}^{\%} \mathrm{AgSbF}_{6}$ & $\mathrm{CH}_{3} \mathrm{OH}, 80{ }^{\circ} \mathrm{C}$ & 57 \\
\hline 4 & $10 \mathrm{~mol}^{\%} \mathrm{AgSbF}_{6}$ & $\mathrm{CH}_{3} \mathrm{OH}, 100{ }^{\circ} \mathrm{C}$ & 79 \\
\hline 5 & $10 \mathrm{~mol}^{\%} \mathrm{AgSbF}_{6}$ & $\mathrm{CH}_{3} \mathrm{OH}, 110{ }^{\circ} \mathrm{C}$ & 87 \\
\hline 6 & $10 \mathrm{~mol}^{\%} \mathrm{AgSbF}_{6}$ & $\mathrm{CH}_{3} \mathrm{OH}, 120{ }^{\circ} \mathrm{C}$ & 87 \\
\hline 7 & $10 \mathrm{~mol} \% \mathrm{AgCl}$ & $\mathrm{CH}_{3} \mathrm{OH}, 110{ }^{\circ} \mathrm{C}$ & 80 \\
\hline 8 & $10 \mathrm{~mol} \% \mathrm{AgNO}_{3}$ & $\mathrm{CH}_{3} \mathrm{OH}, 110{ }^{\circ} \mathrm{C}$ & 94 \\
\hline 9 & $10 \mathrm{~mol} \%$ AgOTf & $\mathrm{CH}_{3} \mathrm{OH}, 110{ }^{\circ} \mathrm{C}$ & 90 \\
\hline $10^{d}$ & $10 \mathrm{~mol} \% \mathrm{AgOAc}$ & $\mathrm{CH}_{3} \mathrm{OH}, 110{ }^{\circ} \mathrm{C}$ & 98 \\
\hline 11 & $10 \mathrm{~mol} \% \mathrm{Ag}_{2} \mathrm{SO}_{4}$ & $\mathrm{CH}_{3} \mathrm{OH}, 110{ }^{\circ} \mathrm{C}$ & 87 \\
\hline 12 & $10 \mathrm{~mol} \% \mathrm{AgBF}_{4}$ & $\mathrm{CH}_{3} \mathrm{OH}, 110{ }^{\circ} \mathrm{C}$ & 84 \\
\hline 13 & $10 \mathrm{~mol}^{\circ} \mathrm{AgPF}_{6}$ & $\mathrm{CH}_{3} \mathrm{OH}, 110{ }^{\circ} \mathrm{C}$ & 81 \\
\hline 14 & $15 \%$ mol AgOAc & $\mathrm{CH}_{3} \mathrm{OH}, 110{ }^{\circ} \mathrm{C}$ & 98 \\
\hline 15 & $5 \% \mathrm{~mol} \mathrm{AgOAc}$ & $\mathrm{CH}_{3} \mathrm{OH}, 110{ }^{\circ} \mathrm{C}$ & 87 \\
\hline 16 & $10 \mathrm{~mol} \% \mathrm{AgOAc}$ & $\mathrm{H}_{2} \mathrm{O}, 110{ }^{\circ} \mathrm{C}$ & 9 \\
\hline 17 & $10 \mathrm{~mol} \% \mathrm{AgOAc}$ & $\mathrm{DCM}, 110{ }^{\circ} \mathrm{C}$ & 15 \\
\hline 18 & $10 \mathrm{~mol} \% \mathrm{AgOAc}$ & $\mathrm{CH}_{3} \mathrm{CN} 110{ }^{\circ} \mathrm{C}$ & 12 \\
\hline 19 & $10 \mathrm{~mol} \% \mathrm{AgOAc}$ & $\mathrm{THF}, 110{ }^{\circ} \mathrm{C}$ & 25 \\
\hline 20 & $10 \mathrm{~mol} \% \mathrm{AgOAc}$ & $\mathrm{EtOH}, 110{ }^{\circ} \mathrm{C}$ & 50 \\
\hline 21 & $10 \mathrm{~mol} \% \mathrm{Fe}(\mathrm{OAc})_{3}$ & $\mathrm{CH}_{3} \mathrm{OH}, 110{ }^{\circ} \mathrm{C}$ & - \\
\hline 22 & $10 \mathrm{~mol} \% \mathrm{Cu}(\mathrm{OAc})_{2}$ & $\mathrm{CH}_{3} \mathrm{OH}, 110{ }^{\circ} \mathrm{C}$ & - \\
\hline 23 & $10 \mathrm{~mol} \% \mathrm{H}_{2} \mathrm{SO}_{4}$ & $\mathrm{CH}_{3} \mathrm{OH}, 110{ }^{\circ} \mathrm{C}$ & - \\
\hline 24 & $10 \mathrm{~mol} \% \mathrm{HOAc}$ & $\mathrm{CH}_{3} \mathrm{OH}, 110{ }^{\circ} \mathrm{C}$ & - \\
\hline $25^{e}$ & $10 \mathrm{~mol} \% \mathrm{AgOAc}$ & $\mathrm{CH}_{3} \mathrm{OH}, 110{ }^{\circ} \mathrm{C}$ & 92 \\
\hline $26^{f}$ & $10 \mathrm{~mol} \% \mathrm{AgOAc}$ & $\mathrm{CH}_{3} \mathrm{OH}, 110{ }^{\circ} \mathrm{C}$ & 94 \\
\hline $27^{g}$ & $10 \mathrm{~mol} \% \mathrm{AgOAc}$ & $\mathrm{CH}_{3} \mathrm{OH}, 110{ }^{\circ} \mathrm{C}$ & 96 \\
\hline
\end{tabular}

${ }^{a}$ Alkynylphosphonate 1a $(0.3 \mathrm{mmol}), \mathrm{H}_{2} \mathrm{O}(1.2 \mathrm{mmol})$, silver catalyst $(10 \mathrm{~mol} \%)$, methanol $(3 \mathrm{~mL})$ in the seal tube. ${ }^{b}$ The reaction progress was monitored by Thin Layer Chromatography. ${ }^{c}$ Estimated by GC. ${ }^{d}$ Isolated yield was $96 \% .{ }^{e}$ Under $\mathrm{O}_{2} .{ }^{f}$ Under $\mathrm{N}_{2} .{ }^{g}$ Alkynylphosphonate 1a (5 mmol, $\left.1.46 \mathrm{~g}\right), \mathrm{H}_{2} \mathrm{O}(20 \mathrm{mmol})$, silver catalyst $(10 \mathrm{~mol} \%)$, methanol $(50 \mathrm{~mL})$ in the seal tube. 
件: 炔基膦酸酯(0.3 mmol), 水(1.2 mmol), AgOAc (0.03 $\mathrm{mmol}), 3 \mathrm{~mL}$ 甲醇, $110{ }^{\circ} \mathrm{C}$ 搅拌反应 $24 \mathrm{~h}$. 在最优条件下, 我们还在氧气或氮气氛围下进行了反应(Table 1, Entries $25,26)$, 实验证明, 收率基本不变, 这也从侧面证明了 水合反应中氧来源于水. 在最优条件下, 作者还进了放 大实验(Table 1, Entry 27), 结果表明收率基本不变.

\section{2 底物范围扩展}

在最佳反应条件下, 作者对多种炔基膦酸酯的水合 反应进行了研究, 结果显示于 Table 2. 当芳炔基膦酸酯 底物芳环的对位、间位存在供电子基团(甲基和甲氧基) 或吸电子基团(溴和三氟甲基)时, 底物都能近乎完全转 化成相应的 $\beta$-羰基膦酸酯化合物(Table 2 , Entries 1 7). 在芳炔底物的邻位有甲基或溴原子时, 反应也能非常顺
利的进行(Table 2, Entries 8,9). 此外具有生物活性的杂 环底物，如噻吩、䒺、胡椒基等都能高产率的得到相应 的水合产物(Table 2, Entries 10 12). 脂肪烃底物的研 究结果表明, 该反应能很好的兼容含多种重要官能团的 炔基膦酸酯，包括卤素(Table 2, Entry 13)、硅醚(Table 2, Entry 14)、乙酰氧基(Table 2, Entry 15)、磺酸酯(Table 2, Entry 16)、邻苯二甲酰亚胺(Table 2, Entry 17)、磺酰胺 (Table 2, Entry 18)、环已烯基(Table 2, Entry 19)和环丙基 (Table 2, Entry 20). 研究还发现其他的膦酸酯底物, 如 二甲氧基、二异丙氧基、二正丁氧基和二苄氧基(Table 2, Entires 21 24)都能顺利的发生水合反应，高产率的得 到相应目标化合物. 当使用中间炔基膦酸酯化合物进行 反应时发现没有检测到产物(Table 2, Entry 25).

表 2 反应范围 ${ }^{a}$

Table 2 The reaction scope

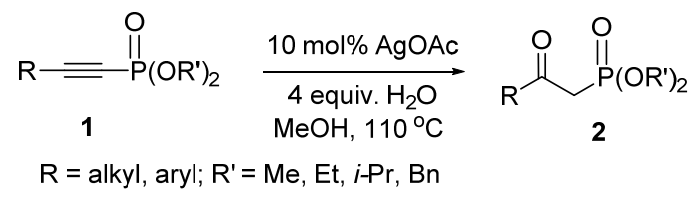

\begin{tabular}{|c|c|c|c|c|c|}
\hline Entry & Product & Yield $/ \%$ & Entry & Product & Yield $^{b} / \%$ \\
\hline 1 & $2 b$ & 95 & 2 & $2 c$ & 96 \\
\hline 3 & $2 d$ & 94 & 4 & $2 e$ & 98 \\
\hline 5 & $2 \mathrm{f}$ & 97 & 6 & $2 \mathrm{~g}$ & 97 \\
\hline 7 & $2 \mathrm{~h}$ & 94 & 8 & $2 i$ & 93 \\
\hline 9 & $2 \mathbf{j}$ & 94 & 10 & $2 k$ & 92 \\
\hline
\end{tabular}




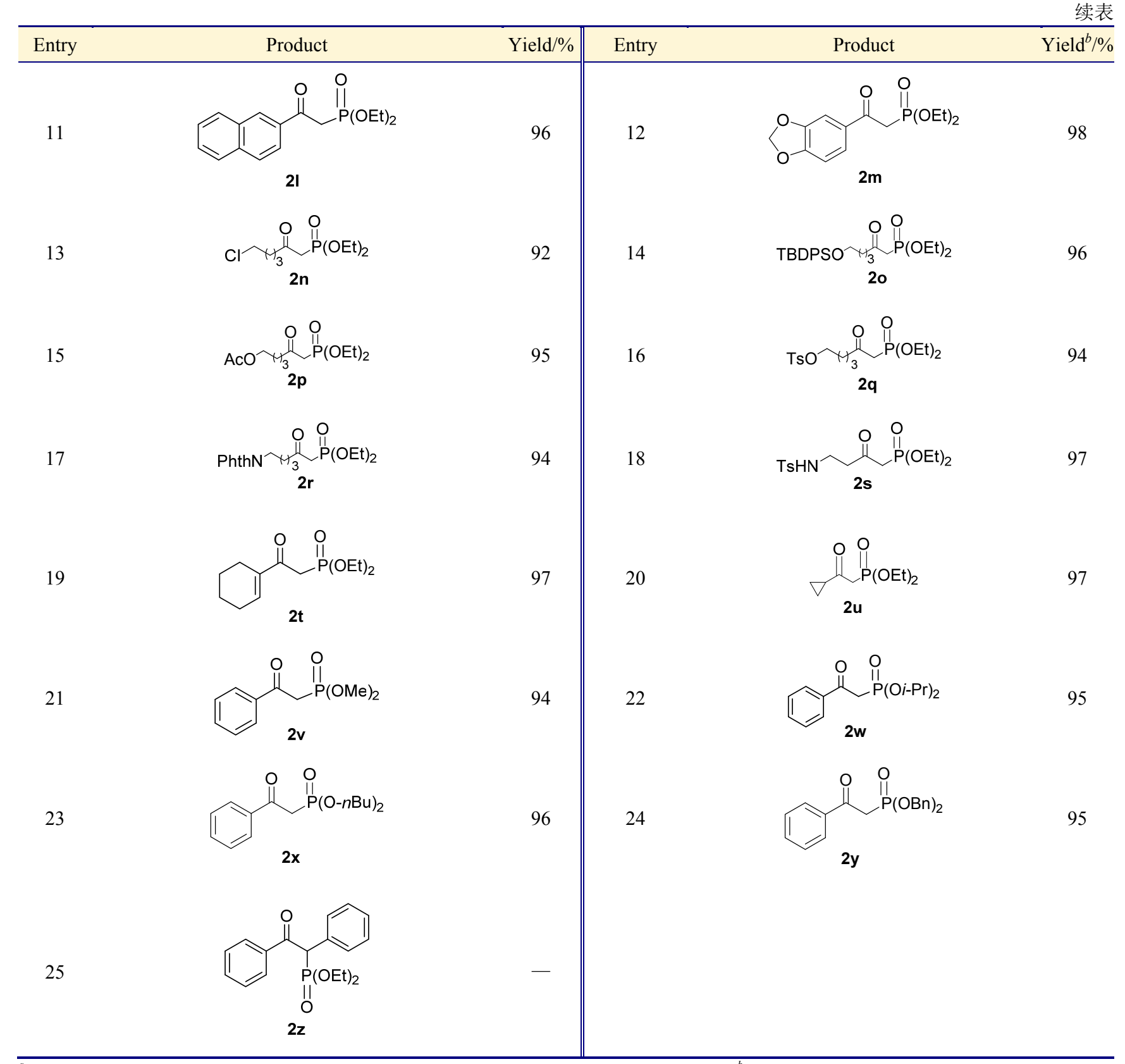

${ }^{a}$ Alkynylphosphonate $1(0.3 \mathrm{mmol}), \mathrm{H}_{2} \mathrm{O}(1.2 \mathrm{mmol}), \mathrm{AgOAc}(10 \mathrm{~mol} \%)$, Methanol $(3 \mathrm{~mL})$ in the seal tube. ${ }^{b}$ Isolated yield.

\section{3 机理研究}

机理研究中, 本文通过 $\mathrm{H}_{2}{ }^{18} \mathrm{O}$ 实验进一步证实了 $\beta$ 羰基膦酸酯化合物的氧来源于水(Eq. 1).

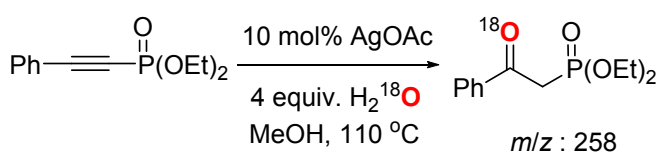

在 Leyva 等报道的金/甲醇 ${ }^{[29]}$ 、银/甲醇 ${ }^{[30]}$ 促进的水 合反应基础上, 结合本文的实验数据, 作者提出以下反 应机理(Scheme 1). 首先银离子活化炔基膦酸酯, 然后 甲醇进攻银活化的炔基形成配位过渡态 $\mathbf{A}$, 接着 $\mathbf{A}$ 发生 质子去银化生成烯醇中间体 $\mathbf{B}$, 中间体 $\mathbf{B}$ 容易与甲醇进
一步加成形成缩酮中间体 $\mathbf{C}$, 中间体 $\mathbf{B}$ 和 $\mathbf{C}$ 存在着动态 平衡; 最后水分子进攻中间体 $\mathbf{B}$ 和 $\mathbf{C}$, 得到 $\beta$-羰基膦酸 酯化合物. 作者尝试分离中间体 $\mathbf{B}$ 和 $\mathbf{C}$, 没有成功.

\section{2 结论}

我们研究了醋酸银/甲醇促进的炔基膦酸酯化合物 水合反应, 开发了一种操作简便、原子经济性高的合成 $\beta$-羰基膦酸酯化合物的新方法. 甲醇在反应中不仅作为 溶剂, 还发挥着助催化剂的作用. 该方法无需加入酸性 或碱性助催化剂、无需惰性气体保护，不使用、不产生 有毒危害试剂, 反应条件温和且具有广泛的反应底物普 适性。该研究工作不仅丰富了银催化炔烃水合反应体 


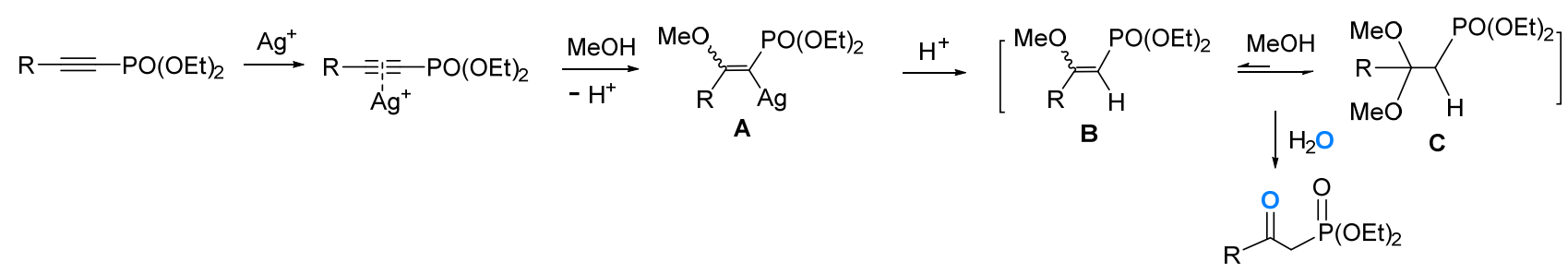

图 1 可能反应机理

Scheme 1 Possible reaction mechanism

系, 并为含有 $\beta$-羰基膦酸酯结构的天然产物及药物分子 合成提供了新的途径.

\section{3 实验部分}

\section{1 试剂与仪器}

${ }^{1} \mathrm{H} \mathrm{NMR}$ 和 ${ }^{13} \mathrm{C} \mathrm{NMR}$ (内标为 $\mathrm{TMS}$, 溶剂为 $\mathrm{CDCl}_{3}$ ) 使用 Varian INOVA-400 型核磁共振仪测定; GC 使用岛 津 GC-2010气相色谱仪测定; 薄层和柱层析用硅胶均为 青岛海洋化工厂产品. 实验所用试剂均为国产分析纯试 剂.

\section{2 银催化炔基膦酸酯水合反应}

在 $10 \mathrm{~mL}$ 密封管中依次加入 $0.3 \mathrm{mmol}$ 炔基膦酸酯 化合物 1、3 mL 甲醇、 $1.2 \mathrm{mmol}$ 水和 $10 \mathrm{~mol} \% \mathrm{AgOAc}$ $(0.03 \mathrm{mmol})$, 于 $110{ }^{\circ} \mathrm{C}$ 反应 $24 \mathrm{~h}$. 减压旋去溶剂, 得到 粘稠液或固体, 混合物再经柱层析 $[V$ (乙酸乙酯) : $V$ (石 油醚) $=1: 1 \sim 1: 15$ ]分离提纯得目标产物 $\mathbf{2 a} \sim \mathbf{2 z}$.

2 -氧代癸基膦酸二乙酯 $(\mathbf{2 a})^{[20]}$ : 淡黄色油状物. ${ }^{1} \mathrm{H}$ NMR (400 MHz, $\left.\mathrm{CDCl}_{3}\right) \delta: 4.17 \sim 4.09$ (m, 4H), 3.05 (d, $\left.J_{\mathrm{H}-\mathrm{P}}=22.4 \mathrm{~Hz}, 2 \mathrm{H}\right), 2.58(\mathrm{t}, J=7.2 \mathrm{~Hz}, 2 \mathrm{H}), 1.56(\mathrm{t}, J=7.2$ $\mathrm{Hz}, 2 \mathrm{H}), 1.32 \sim 1.23(\mathrm{~m}, 16 \mathrm{H}), 0.86(\mathrm{t}, J=7.2 \mathrm{~Hz}, 3 \mathrm{H}) ;{ }^{13} \mathrm{C}$ NMR (100 MHz, $\left.\mathrm{CDCl}_{3}\right) \delta: 202.21\left(\mathrm{~d}, J_{\mathrm{C}-\mathrm{P}}=5.9 \mathrm{~Hz}\right)$, $62.43\left(\mathrm{~d}, J_{\mathrm{C}-\mathrm{P}}=6.5 \mathrm{~Hz}\right), 44.04,42.24\left(\mathrm{~d}, J_{\mathrm{C}-\mathrm{P}}=126.1 \mathrm{~Hz}\right)$, $31.72,29.25,29.03,28.87,23.35,22.53,16.23\left(\mathrm{~d}, J_{\mathrm{C}-\mathrm{P}}=\right.$ $6.6 \mathrm{~Hz}$ ), 13.98; HRMS (ESI-TOF) calcd for $\mathrm{C}_{14} \mathrm{H}_{29} \mathrm{O}_{4} \mathrm{P}$ $[\mathrm{M}+\mathrm{H}]^{+}$292.1802, found 292.1798.

(2-苯乙基-2-氧代)膦酸二乙酯 $(\mathbf{2 b})^{[20]}$ : 淡黄色油状 物. ${ }^{1} \mathrm{H}$ NMR (400 MHz, $\left.\mathrm{CDCl}_{3}\right) \delta: 8.01(\mathrm{~d}, J=8.1 \mathrm{~Hz}$, 2H), 7.59 (t, $J=7.4 \mathrm{~Hz}, 1 \mathrm{H}), 7.48$ (t, $J=7.7 \mathrm{~Hz}, 2 \mathrm{H})$, $4.18 \sim 4.12(\mathrm{~m}, 4 \mathrm{H}), 3.64(\mathrm{~d}, J=22.7 \mathrm{~Hz}, 2 \mathrm{H}), 1.27(\mathrm{t}, J=$ $7.1 \mathrm{~Hz}, 6 \mathrm{H}) ;{ }^{13} \mathrm{C}$ NMR (100 MHz, $\left.\mathrm{CDCl}_{3}\right) \delta: 191.9(\mathrm{~d}$, $\left.J_{\mathrm{C}-\mathrm{P}}=6.8 \mathrm{~Hz}\right), 136.7,133.5,129.1,128.7,62.7\left(\mathrm{~d}, J_{\mathrm{C}-\mathrm{P}}=\right.$ $5.7 \mathrm{~Hz}), 38.6\left(\mathrm{~d}, J_{\mathrm{C}-\mathrm{P}}=128.9 \mathrm{~Hz}\right), 16.1\left(\mathrm{~d}, J_{\mathrm{C}-\mathrm{P}}=5.7 \mathrm{~Hz}\right)$; HRMS (ESI-TOF) calcd for $\mathrm{C}_{12} \mathrm{H}_{17} \mathrm{O}_{4} \mathrm{P} \quad[\mathrm{M}+\mathrm{H}]{ }^{+}$ 256.0865, found 256.0862 .

[2-(4'-甲基苯乙基)-2-氧代]膦酸二乙酯(2c) ${ }^{[31]}$ : 淡 黄色油状物. ${ }^{1} \mathrm{H}$ NMR (400 $\left.\mathrm{MHz}, \mathrm{CDCl}_{3}\right) \delta: 7.86(\mathrm{~d}, J=$
$8.0 \mathrm{~Hz}, 2 \mathrm{H}), 7.24(\mathrm{t}, J=8.0 \mathrm{~Hz}, 2 \mathrm{H}), 4.05 \sim 4.13(\mathrm{~m}, 4 \mathrm{H})$, $3.56\left(\mathrm{~d}, J_{\mathrm{H}-\mathrm{P}}=22.4 \mathrm{~Hz}, 2 \mathrm{H}\right), 2.36(\mathrm{~s}, 3 \mathrm{H}), 1.23(\mathrm{t}, J=7.2$ $\mathrm{Hz}, 6 \mathrm{H}) ;{ }^{13} \mathrm{C}$ NMR $\left(100 \mathrm{MHz}, \mathrm{CDCl}_{3}\right) \delta: 191.38\left(\mathrm{~d}, J_{\mathrm{C}-\mathrm{P}}=\right.$ $6.5 \mathrm{~Hz}), 144.53,133.93,129.18,129.06,62.48\left(\mathrm{~d}, J_{\mathrm{C}-\mathrm{P}}=\right.$ $6.5 \mathrm{~Hz}), 38.22$ (d, $\left.J_{\mathrm{C}-\mathrm{P}}=129.0 \mathrm{~Hz}\right), 21.57,16.13\left(\mathrm{~d}, J_{\mathrm{C}-\mathrm{P}}=\right.$ $6.5 \mathrm{~Hz}$ ); HRMS (ESI-TOF) calcd for $\mathrm{C}_{13} \mathrm{H}_{19} \mathrm{O}_{4} \mathrm{P}[\mathrm{M}+\mathrm{H}]^{+}$ 508.1780, found 508.1776.

[2-(4'-甲氧基苯乙基)-2-氧代]膦酸二乙酯 $(2 d)^{[31]}$ : 淡黄色油状物. ${ }^{1} \mathrm{H}$ NMR (400 $\left.\mathrm{MHz}, \mathrm{CDCl}_{3}\right) \delta: 7.98(\mathrm{~d}$, $J=8.8 \mathrm{~Hz}, 2 \mathrm{H}), 6.93(\mathrm{~d}, J=8.8 \mathrm{~Hz}, 2 \mathrm{H}), 4.14 \sim 4.07(\mathrm{~m}$, $4 \mathrm{H}), 3.84(\mathrm{~s}, 3 \mathrm{H}), 3.57\left(\mathrm{~d}, J_{\mathrm{H}-\mathrm{P}}=22.8 \mathrm{~Hz}, 2 \mathrm{H}\right), 1.27(\mathrm{t}$, $\left.J_{\mathrm{C}-\mathrm{P}}=7.2 \mathrm{~Hz}, 6 \mathrm{H}\right) ;{ }^{13} \mathrm{C} \mathrm{NMR}\left(100 \mathrm{MHz}, \mathrm{CDCl}_{3}\right) \delta: 190.18$ $\left(\mathrm{d}, J_{\mathrm{C}-\mathrm{P}}=6.5 \mathrm{~Hz}\right), 163.95,131.44,129.58,113.72,62.61$ $\left(\mathrm{d}, J_{\mathrm{C}-\mathrm{P}}=6.6 \mathrm{~Hz}\right), 55.46,38.14\left(\mathrm{~d}, J_{\mathrm{C}-\mathrm{P}}=129.1 \mathrm{~Hz}\right), 16.17$ $\left(\mathrm{d}, J_{\mathrm{C}-\mathrm{P}}=6.6 \mathrm{~Hz}\right)$; HRMS (ESI-TOF) calcd for $\mathrm{C}_{13} \mathrm{H}_{19} \mathrm{O}_{5} \mathrm{P}$ $[\mathrm{M}+\mathrm{H}]^{+}$286.0971, found 286.0964.

[2-(4'-溴苯乙基)-2-氧代]膦酸二乙酯 $(2 \mathbf{e})^{[32]}$ : 淡黄 色油状物. ${ }^{1} \mathrm{H}$ NMR $\left(400 \mathrm{MHz}, \mathrm{CDCl}_{3}\right) \delta: 7.87(\mathrm{dd}, J=$ $8.4,1.2 \mathrm{~Hz}, 2 \mathrm{H}), 7.61(\mathrm{dd}, J=8.4,1.2 \mathrm{~Hz}, 2 \mathrm{H}), 4.12 \sim 4.08$ $(\mathrm{m}, 4 \mathrm{H}), 3.56\left(\mathrm{~d}, J_{\mathrm{H}-\mathrm{P}}=22.4 \mathrm{~Hz}, 2 \mathrm{H}\right), 1.26(\mathrm{t}, J=7.2 \mathrm{~Hz}$, $6 \mathrm{H}) ;{ }^{13} \mathrm{C} \mathrm{NMR}\left(100 \mathrm{MHz}, \mathrm{CDCl}_{3}\right) \delta: 190.89\left(\mathrm{~d}, J_{\mathrm{C}-\mathrm{P}}=6.6\right.$ $\mathrm{Hz}), 135.18,131.89,130.53,129.01,62.70\left(\mathrm{~d}, J_{\mathrm{C}-\mathrm{P}}=6.6\right.$ $\mathrm{Hz}), 38.57$ (d, $\left.J_{\mathrm{C}-\mathrm{P}}=128.3 \mathrm{~Hz}\right), 16.19\left(\mathrm{~d}, J_{\mathrm{C}-\mathrm{P}}=6.6 \mathrm{~Hz}\right)$; HRMS (ESI-TOF) calcd for $\mathrm{C}_{12} \mathrm{H}_{16} \mathrm{BrO}_{4} \mathrm{P}[\mathrm{M}+\mathrm{H}]^{+}$ 333.9970 , found 333.9962 .

[2-(4'-三氟甲基苯乙基)-2-氧代]膦酸二乙酯 $(2 \mathbf{f})^{[33]}$ : 淡黄色油状物. ${ }^{1} \mathrm{H}$ NMR (400 $\left.\mathrm{MHz}, \mathrm{CDCl}_{3}\right) \delta: 8.11(\mathrm{~d}$, $J=8.0 \mathrm{~Hz}, 2 \mathrm{H}), 7.71(\mathrm{~d}, J=8.0 \mathrm{~Hz}, 2 \mathrm{H}), 4.15 \sim 4.08(\mathrm{~m}$, $4 \mathrm{H}), 3.63\left(\mathrm{~d}, J_{\mathrm{H}-\mathrm{P}}=23.2 \mathrm{~Hz}, 2 \mathrm{H}\right), 1.26(\mathrm{t}, J=7.2 \mathrm{~Hz}, 6 \mathrm{H})$; ${ }^{13} \mathrm{C}$ NMR $\left(100 \mathrm{MHz}, \mathrm{CDCl}_{3}\right) \delta: 191.02\left(\mathrm{~d}, J_{\mathrm{C}-\mathrm{P}}=6.5 \mathrm{~Hz}\right)$, $138.98,134.69\left(\mathrm{q}, J_{\mathrm{C}-\mathrm{F}}=32.8 \mathrm{~Hz}\right), 129.32,125.53(\mathrm{q}$, $\left.J_{\mathrm{C}-\mathrm{F}}=3.7 \mathrm{~Hz}\right), 122.04,62.72\left(\mathrm{~d}, J_{\mathrm{C}-\mathrm{P}}=6.5 \mathrm{~Hz}\right), 38.78(\mathrm{~d}$, $\left.J_{\mathrm{C}-\mathrm{P}}=128.3 \mathrm{~Hz}\right), 16.08\left(\mathrm{~d}, J_{\mathrm{C}-\mathrm{P}}=6.5 \mathrm{~Hz}\right)$; HRMS (ESI-TOF) calcd for $\mathrm{C}_{13} \mathrm{H}_{16} \mathrm{~F}_{3} \mathrm{O}_{4} \mathrm{P}[\mathrm{M}+\mathrm{H}]^{+}$324.9973, found 333.9961 .

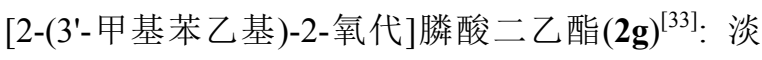
黄色油状物. ${ }^{1} \mathrm{H} \mathrm{NMR}\left(400 \mathrm{MHz}, \mathrm{CDCl}_{3}\right) \delta: 7.77 \sim 7.75$ 
(m, 2H), $7.35 \sim 7.28(\mathrm{~m}, 2 \mathrm{H}), 4.13 \sim 4.06(\mathrm{~m}, 4 \mathrm{H}), 3.58(\mathrm{~d}$, $\left.J_{\mathrm{H}-\mathrm{P}}=22.8 \mathrm{~Hz}, 2 \mathrm{H}\right), 2.36(\mathrm{~s}, 3 \mathrm{H}), 1.24(\mathrm{t}, J=7.2 \mathrm{~Hz}, 6 \mathrm{H})$; ${ }^{13} \mathrm{C}$ NMR $\left(100 \mathrm{MHz}, \mathrm{CDCl}_{3}\right) \delta: 191.98\left(\mathrm{~d}, J_{\mathrm{C}-\mathrm{P}}=6.5 \mathrm{~Hz}\right)$, $138.27,136.45,134.32,129.32,128.36,126.22,62.52(\mathrm{~d}$, $\left.J_{\mathrm{C}-\mathrm{P}}=6.6 \mathrm{~Hz}\right), 38.28\left(\mathrm{~d}, J_{\mathrm{C}-\mathrm{P}}=129.8 \mathrm{~Hz}\right), 21.16,16.09(\mathrm{~d}$, $J_{\text {C-P }}=6.6 \mathrm{~Hz}$ ); HRMS (ESI-TOF) calcd for $\mathrm{C}_{13} \mathrm{H}_{19} \mathrm{O}_{4} \mathrm{P}$ $[\mathrm{M}+\mathrm{H}]^{+} 270.1022$, found 270.1013 .

[2-(3'-溴苯乙基)-2-氧代]膦酸二乙酯 $(\mathbf{2 h})^{[26]}$ : 淡黄 色油状物. ${ }^{1} \mathrm{H}$ NMR $\left(400 \mathrm{MHz}, \mathrm{CDCl}_{3}\right) \delta: 8.12(\mathrm{t}, J=2.0$ $\mathrm{Hz}, 1 \mathrm{H}), 7.93(\mathrm{dt}, J=8.0 \mathrm{~Hz}, 1.2 \mathrm{~Hz}, 1 \mathrm{H}), 7.70 \sim 7.67(\mathrm{~m}$, $1 \mathrm{H}), 7.32$ (t, $J=8.0 \mathrm{~Hz}, 1 \mathrm{H}), 4.13 \sim 4.05(\mathrm{~m}, 4 \mathrm{H}), 3.58$ (d, $\left.J_{\mathrm{H}-\mathrm{P}}=22.4 \mathrm{~Hz}, 2 \mathrm{H}\right), 1.26(\mathrm{t}, J=7.2 \mathrm{~Hz}, 6 \mathrm{H}) ;{ }^{13} \mathrm{C}$ NMR $\left(100 \mathrm{MHz}, \mathrm{CDCl}_{3}\right) \delta: 190.55\left(\mathrm{~d}, J_{\mathrm{C}-\mathrm{P}}=6.5 \mathrm{~Hz}\right), 138.09$, $136.36,131.88,130.08,127.56,122.81,62.66\left(\mathrm{~d}, J_{\mathrm{C}-\mathrm{P}}=6.5\right.$ $\mathrm{Hz}), 38.56\left(\mathrm{~d}, J_{\mathrm{C}-\mathrm{P}}=128.3 \mathrm{~Hz}\right), 16.13\left(\mathrm{~d}, J_{\mathrm{C}-\mathrm{P}}=5.8 \mathrm{~Hz}\right)$; HRMS (ESI-TOF) calcd for $\mathrm{C}_{12} \mathrm{H}_{16} \mathrm{BrO}_{4} \mathrm{P}[\mathrm{M}+\mathrm{H}]^{+}$ 333.9971, found 333.9963.

[2-(2'-甲基苯乙基)-2-氧代]膦酸二乙酯 $(2 \mathbf{i})^{[26]}$ : 淡黄 色油状物. ${ }^{1} \mathrm{H}$ NMR (400 MHz, $\left.\mathrm{CDCl}_{3}\right) \delta: 7.66(\mathrm{~d}, J=7.2$ $\mathrm{Hz}, 1 \mathrm{H}), 7.32$ (t, $J=7.4 \mathrm{~Hz}, 1 \mathrm{H}), 7.22 \sim 7.16$ (dd, $J=7.2$ $\mathrm{Hz}, 7.6 \mathrm{~Hz}, 2 \mathrm{H}), 4.08 \sim 4.01(\mathrm{~m}, 4 \mathrm{H}), 3.53\left(\mathrm{~d}, J_{\mathrm{H}-\mathrm{P}}=22.4\right.$ $\mathrm{Hz}, 2 \mathrm{H}), 2.43$ (s, 3H), 1.17 (t, $J=7.0 \mathrm{~Hz}, 6 \mathrm{H}) ;{ }^{13} \mathrm{C} \mathrm{NMR}$ $\left(100 \mathrm{MHz}, \mathrm{CDCl}_{3}\right) \delta: 194.94\left(\mathrm{~d}, J_{\mathrm{C}-\mathrm{P}}=6.6 \mathrm{~Hz}\right), 138.81$, $137.11,131.85,131.81,129.47,125.55,62.36\left(\mathrm{~d}, J_{\mathrm{C}-\mathrm{P}}=5.9\right.$ $\mathrm{Hz}), 40.94\left(\mathrm{~d}, J_{\mathrm{C}-\mathrm{P}}=129.1 \mathrm{~Hz}\right), 21.17,16.11\left(\mathrm{~d}, J_{\mathrm{C}-\mathrm{P}}=6.6\right.$ $\mathrm{Hz}$ ); HRMS (ESI-TOF) calcd for $\mathrm{C}_{13} \mathrm{H}_{19} \mathrm{O}_{4} \mathrm{P}[\mathrm{M}+\mathrm{H}]^{+}$ 270.1021, found 270.1012 .

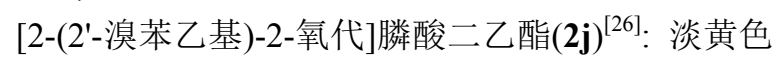
油状物. ${ }^{1} \mathrm{H}$ NMR $\left(400 \mathrm{MHz}, \mathrm{CDCl}_{3}\right) \delta: 8.11(\mathrm{t}, J=2.0 \mathrm{~Hz}$, $1 \mathrm{H}), 7.93(\mathrm{dt}, J=8.0,1.2 \mathrm{~Hz}, 1 \mathrm{H}), 7.70 \sim 7.67(\mathrm{~m}, 1 \mathrm{H})$, $7.33(\mathrm{t}, J=8.0 \mathrm{~Hz}, 1 \mathrm{H}), 4.14 \sim 4.06(\mathrm{~m}, 4 \mathrm{H}), 3.57(\mathrm{~d}$, $\left.J_{\mathrm{H}-\mathrm{P}}=22.4 \mathrm{~Hz}, 2 \mathrm{H}\right), 1.26(\mathrm{t}, J=7.2 \mathrm{~Hz}, 6 \mathrm{H}) ;{ }^{13} \mathrm{C} \mathrm{NMR}$ $\left(100 \mathrm{MHz}, \mathrm{CDCl}_{3}\right) \delta: 190.55\left(\mathrm{~d}, J_{\mathrm{C}-\mathrm{P}}=6.5 \mathrm{~Hz}\right), 138.09$, $136.36,131.88,130.08,127.56,121.81,62.66\left(\mathrm{~d}, J_{\mathrm{C}-\mathrm{P}}=6.5\right.$ $\mathrm{Hz}), 38.56\left(\mathrm{~d}, J_{\mathrm{C}-\mathrm{P}}=128.3 \mathrm{~Hz}\right), 16.13\left(\mathrm{~d}, J_{\mathrm{C}-\mathrm{P}}=5.8 \mathrm{~Hz}\right)$; HRMS (ESI-TOF) calcd for $\mathrm{C}_{12} \mathrm{H}_{16} \mathrm{BrO}_{4} \mathrm{P}[\mathrm{M}+\mathrm{H}]^{+}$ 333.9971, found 333.9964.

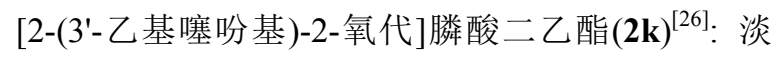
黄色油状物. ${ }^{1} \mathrm{H}$ NMR (400 MHz, $\left.\mathrm{CDCl}_{3}\right){ }^{1} \mathrm{H}$ NMR (400 $\left.\mathrm{MHz}, \mathrm{CDCl}_{3}\right) \delta: 8.14(\mathrm{~d}, J=2.0 \mathrm{~Hz}, 1 \mathrm{H}), 7.52(\mathrm{~d}, J=6.1$ $\mathrm{Hz}, 1 \mathrm{H}), 7.25 \sim 7.24(\mathrm{~m}, 1 \mathrm{H}), 4.11 \sim 4.04(\mathrm{~m}, 4 \mathrm{H}), 3.45(\mathrm{~d}$, $\left.J_{\mathrm{H}-\mathrm{P}}=22.8 \mathrm{~Hz}, 2 \mathrm{H}\right), 1.23(\mathrm{t}, J=7.2 \mathrm{~Hz}, 6 \mathrm{H}) ;{ }^{13} \mathrm{C} \mathrm{NMR}$ $\left(100 \mathrm{MHz}, \mathrm{CDCl}_{3}\right) \delta: 185.49\left(\mathrm{~d}, J_{\mathrm{C}-\mathrm{P}}=6.6 \mathrm{~Hz}\right), 141.78$, $134.26,127.18,126.30,62.58\left(\mathrm{~d}, J_{\mathrm{C}-\mathrm{P}}=6.5 \mathrm{~Hz}\right), 39.89(\mathrm{~d}$, $\left.J_{\mathrm{C}-\mathrm{P}}=128.4 \mathrm{~Hz}\right), 16.12\left(\mathrm{~d}, J_{\mathrm{C}-\mathrm{P}}=6.6 \mathrm{~Hz}\right) ; \mathrm{HRMS}$
(ESI-TOF) calcd for $\mathrm{C}_{10} \mathrm{H}_{15} \mathrm{O}_{4} \mathrm{PS}[\mathrm{M}+\mathrm{H}]^{+}$262.0429, found 262.0422 .

(2-菜乙基-2-氧代)膦酸二乙酯 $(21)^{[33]}$ : 淡黄色油状 物. ${ }^{1} \mathrm{H}$ NMR (400 MHz, $\mathrm{CDCl}_{3}$ ) $\delta: 8.48$ (s, 1H), 7.97 (dd, $J=1.6,8.4 \mathrm{~Hz}, 1 \mathrm{H}), 7.91(\mathrm{~d}, J=8.0 \mathrm{~Hz}, 1 \mathrm{H}), 7.80$ (t, $J=$ $9.0 \mathrm{~Hz}, 2 \mathrm{H}), 7.53 \sim 7.47(\mathrm{~m}, J=7.5 \mathrm{~Hz}, 2 \mathrm{H}), 4.09 \sim 4.06$ (m, 4H), $3.69(\mathrm{~d}, J=22.7 \mathrm{~Hz}, 2 \mathrm{H}), 1.20(\mathrm{t}, J=7.2 \mathrm{~Hz}, 6 \mathrm{H}$, overlap); ${ }^{13} \mathrm{C}$ NMR $\left(100 \mathrm{MHz}, \mathrm{CDCl}_{3}\right) \delta: 191.8\left(\mathrm{~d}, J_{\mathrm{C}-\mathrm{P}}=\right.$ $5.7 \mathrm{~Hz}), 135.6,134.2,132.5,131.6,129.7,128.8,128.3$, $127.8,126.9,124.2,71.4\left(\mathrm{~d}, J_{\mathrm{C}-\mathrm{P}}=5.7 \mathrm{~Hz}\right), 39.8\left(\mathrm{~d}, J_{\mathrm{C}-\mathrm{P}}=\right.$ $130.0 \mathrm{~Hz}), 23.8\left(\mathrm{~d}, J_{\mathrm{C}-\mathrm{P}}=3.4 \mathrm{~Hz}\right), 23.9\left(\mathrm{~d}, J_{\mathrm{C}-\mathrm{P}}=4.6 \mathrm{~Hz}\right)$; HRMS (EI): calcd for $\mathrm{C}_{16} \mathrm{H}_{19} \mathrm{O}_{4} \mathrm{P}[\mathrm{M}]^{+}$306.1022, found: 306.1017.

[2-(3',4'-亚甲二氧基苯乙基)-2-氧代]膦酸二乙酯 $(2 m)^{[26]}$ : 淡黄色油状物. ${ }^{1} \mathrm{H}$ NMR $\left(400 \mathrm{MHz}, \mathrm{CDCl}_{3}\right) \delta$ : 7.58 (d, $J=8.4 \mathrm{~Hz}, 1 \mathrm{H}), 7.42$ (s, 1H), 6.83 (d, $J=8.4 \mathrm{~Hz}$, $1 \mathrm{H}), 6.02(\mathrm{~s}, 2 \mathrm{H}), 4.15 \sim 4.07(\mathrm{~m}, 4 \mathrm{H}), 3.53\left(\mathrm{~d}, J_{\mathrm{H}-\mathrm{P}}=22.8\right.$ $\mathrm{Hz}, 2 \mathrm{H}), 1.24$ (t, $J=7.2 \mathrm{~Hz}, 6 \mathrm{H}) ;{ }^{13} \mathrm{C}$ NMR $(100 \mathrm{MHz}$, $\left.\mathrm{CDCl}_{3}\right) \delta: 189.72\left(\mathrm{~d}, J_{\mathrm{C}-\mathrm{P}}=6.6 \mathrm{~Hz}\right), 152.26,148.18$, $131.32,125.97,108.36,107.75,101.93,62.58\left(\mathrm{~d}, J_{\mathrm{C}-\mathrm{P}}=6.6\right.$ $\mathrm{Hz}), 38.24\left(\mathrm{~d}, J_{\mathrm{C}-\mathrm{P}}=129.8 \mathrm{~Hz}\right), 16.16\left(\mathrm{~d}, J_{\mathrm{C}-\mathrm{P}}=6.6 \mathrm{~Hz}\right)$; HRMS (ESI-TOF) calcd for $\mathrm{C}_{13} \mathrm{H}_{17} \mathrm{O}_{6} \mathrm{P}[\mathrm{M}+\mathrm{H}]^{+}$ 300.0764 , found 300.0759 .

1-氯-5-氧代已基膦酸二乙酯 (2n): 淡黄色油状物. ${ }^{1} \mathrm{H}$ NMR $\left(400 \mathrm{MHz}, \mathrm{CDCl}_{3}\right) \delta: 4.12 \sim 4.05(\mathrm{~m}, 4 \mathrm{H}), 3.47$ $(\mathrm{t}, J=5.6 \mathrm{~Hz}, 2 \mathrm{H}), 3.02\left(\mathrm{~d}, J_{\mathrm{H}-\mathrm{P}}=22.4 \mathrm{~Hz}, 2 \mathrm{H}\right), 2.63(\mathrm{t}$, $J=6.8 \mathrm{~Hz}, 2 \mathrm{H}), 1.76 \sim 1.63(\mathrm{~m}, 4 \mathrm{H}), 1.28(\mathrm{t}, J=7.2 \mathrm{~Hz}$, $6 \mathrm{H}) ;{ }^{13} \mathrm{C}$ NMR $\left(100 \mathrm{MHz}, \mathrm{CDCl}_{3}\right) \delta: 201.32\left(\mathrm{~d}, J_{\mathrm{C}-\mathrm{P}}=5.9\right.$ $\mathrm{Hz}), 62.51\left(\mathrm{~d}, J_{\mathrm{C}-\mathrm{P}}=6.6 \mathrm{~Hz}\right), 44.44,42.31\left(\mathrm{~d}, J_{\mathrm{C}-\mathrm{P}}=126.2\right.$ $\mathrm{Hz}), 42.82,31.47,20.54,16.17\left(\mathrm{~d}, J_{\mathrm{C}-\mathrm{P}}=6.5 \mathrm{~Hz}\right) ; \mathrm{HRMS}$ (ESI-TOF) calcd for $\mathrm{C}_{10} \mathrm{H}_{20} \mathrm{ClO}_{4} \mathrm{P}[\mathrm{M}+\mathrm{H}]^{+}$270.0786, found 270.0787 .

1-叔丁基二苯基硅烷氧基-5-氧代已基膦酸二乙酯 (2o): 淡黄色油状物. ${ }^{1} \mathrm{H}$ NMR $\left(400 \mathrm{MHz}, \mathrm{CDCl}_{3}\right) \delta$ : $7.65 \sim 7.63(\mathrm{~m}, 4 \mathrm{H}), 7.42 \sim 7.36(\mathrm{~m}, 6 \mathrm{H}), 4.16 \sim 4.11(\mathrm{~m}$, $4 \mathrm{H}), 3.64(\mathrm{t}, J=6.0 \mathrm{~Hz}, 2 \mathrm{H}), 3.05\left(\mathrm{~d}, J_{\mathrm{H}-\mathrm{P}}=22.8 \mathrm{~Hz}, 2 \mathrm{H}\right)$, $2.61(\mathrm{t}, J=7.2 \mathrm{~Hz}, 2 \mathrm{H}), 1.72 \sim 1.65(\mathrm{~m}, 2 \mathrm{H}), 1.58 \sim 1.51$ (m, 2H), 1.33 (t, $J=7.2 \mathrm{~Hz}, 6 \mathrm{H}), 1.05$ (s, 9H); ${ }^{13} \mathrm{C} \mathrm{NMR}$ $\left(100 \mathrm{MHz}, \mathrm{CDCl}_{3}\right) \delta: 201.88\left(\mathrm{~d}, J_{\mathrm{C}-\mathrm{P}}=6.5 \mathrm{~Hz}\right), 135.52$, $133.93,129.52,127.56,63.45,62.48\left(\mathrm{~d}, J_{\mathrm{C}-\mathrm{P}}=6.5 \mathrm{~Hz}\right)$, $42.27\left(\mathrm{~d}, J_{\mathrm{C}-\mathrm{P}}=126.9 \mathrm{~Hz}\right), 31.73,29.63,26.83,19.83$, $19.17,16.25\left(\mathrm{~d}, J_{\mathrm{C}-\mathrm{P}}=6.6 \mathrm{~Hz}\right)$; HRMS (ESI-TOF) calcd for $\mathrm{C}_{26} \mathrm{H}_{39} \mathrm{O}_{5} \mathrm{PSi}[\mathrm{M}+\mathrm{H}]^{+}$490.2303, found 490.2302.

1-乙酰氧基-5-氧代已基膦酸二乙酯 (2p): 淡黄色油 状物. ${ }^{1} \mathrm{H}$ NMR (400 MHz, $\left.\mathrm{CDCl}_{3}\right) \delta: 4.11 \sim 4.08$ (m, 4H), 
$3.98 \sim 3.95(\mathrm{~m}, 2 \mathrm{H}), 3.01\left(\mathrm{~d}, J_{\mathrm{H}-\mathrm{P}}=23.2 \mathrm{~Hz}, 2 \mathrm{H}\right), 2.58(\mathrm{t}$, $J=6.4 \mathrm{~Hz}, 2 \mathrm{H}), 1.95(\mathrm{~s}, 3 \mathrm{H}), 1.58 \sim 1.56(\mathrm{~m}, 4 \mathrm{H}), 1.25(\mathrm{t}$, $J=7.2 \mathrm{~Hz}, 6 \mathrm{H}) ;{ }^{13} \mathrm{C}$ NMR $\left(100 \mathrm{MHz}, \mathrm{CDCl}_{3}\right) \delta: 201.34$ (d, $\left.J_{\mathrm{C}-\mathrm{P}}=6.6 \mathrm{~Hz}\right), 170.92,63.83,62.42\left(\mathrm{~d}, J_{\mathrm{C}-\mathrm{P}}=6.6 \mathrm{~Hz}\right)$, $43.18,42.25\left(\mathrm{~d}, J_{\mathrm{C}-\mathrm{P}}=126.9 \mathrm{~Hz}\right), 27.64,20.75,19.62$, $16.12\left(\mathrm{~d}, J_{\mathrm{C}-\mathrm{P}}=5.8 \mathrm{~Hz}\right.$ ); HRMS (ESI-TOF) calcd for $\mathrm{C}_{12} \mathrm{H}_{23} \mathrm{O}_{6} \mathrm{P}[\mathrm{M}+\mathrm{H}]^{+}$294.1233, found 294.1226.

1-对甲苯磺酰基-5-氧代已基膦酸二乙酯(2q): 淡黄 色油状物. ${ }^{1} \mathrm{H}$ NMR $\left(400 \mathrm{MHz}, \mathrm{CDCl}_{3}\right) \delta: 7.75(\mathrm{~d}, J=8.0$ $\mathrm{Hz}, 2 \mathrm{H}), 7.34(\mathrm{~d}, J=8.0 \mathrm{~Hz}, 2 \mathrm{H}), 4.12 \sim 4.08(\mathrm{~m}, 4 \mathrm{H})$, $4.01(\mathrm{t}, J=6.0 \mathrm{~Hz}, 2 \mathrm{H}), 3.02\left(\mathrm{~d}, J_{\mathrm{H}-\mathrm{P}}=22.8 \mathrm{~Hz}, 2 \mathrm{H}\right), 2.61$ (t, $J=6.8 \mathrm{~Hz}, 2 \mathrm{H}), 2.44$ (s, 3H), $1.66 \sim 1.58(\mathrm{~m}, 4 \mathrm{H}), 1.32$ (t, $J=7.2 \mathrm{~Hz}, 6 \mathrm{H}) ;{ }^{13} \mathrm{C}$ NMR (100 MHz, $\left.\mathrm{CDCl}_{3}\right) \delta: 201.24$ $\left(\mathrm{d}, J_{\mathrm{C}-\mathrm{P}}=5.8 \mathrm{~Hz}\right), 144.75,132.98,129.83,127.83,70.08$, $62.64\left(\mathrm{~d}, J_{\mathrm{C}-\mathrm{P}}=6.6 \mathrm{~Hz}\right), 42.27\left(\mathrm{~d}, J_{\mathrm{C}-\mathrm{P}}=126.1 \mathrm{~Hz}\right), 27.95$, $21.56,19.26,16.22\left(\mathrm{~d}, J_{\mathrm{C}-\mathrm{P}}=5.9 \mathrm{~Hz}\right.$ ); HRMS (ESI-TOF) calcd for $\mathrm{C}_{17} \mathrm{H}_{27} \mathrm{O}_{7} \mathrm{PS}[\mathrm{M}+\mathrm{H}]^{+}$406.1216, found 406.1211.

1-邻苯二甲酰亚胺基-5-氧代已基膦酸二乙酯(2r): 淡黄色油状物. ${ }^{1} \mathrm{H}$ NMR $\left(400 \mathrm{MHz}, \mathrm{CDCl}_{3}\right) \delta: 7.82$ (dd, $J=5.6,2.8 \mathrm{~Hz}, 2 \mathrm{H}), 7.68(\mathrm{dd}, J=5.6,2.8 \mathrm{~Hz}, 2 \mathrm{H}), 4.15 \sim$ 4.08 (m, 4H), 3.66 (t, $J=6.8 \mathrm{~Hz}, 2 \mathrm{H}), 3.04$ (d, $J_{\mathrm{H}-\mathrm{P}}=22.8$ $\mathrm{Hz}, 2 \mathrm{H}), 2.65(\mathrm{t}, J=7.2 \mathrm{~Hz}, 2 \mathrm{H}), 1.70 \sim 1.58(\mathrm{~m}, 4 \mathrm{H}), 1.32$ $(\mathrm{t}, J=7.2 \mathrm{~Hz}, 6 \mathrm{H}) ;{ }^{13} \mathrm{C} \mathrm{NMR}\left(100 \mathrm{Mz}, \mathrm{CDCl}_{3}\right) \delta: 201.38$ $\left(\mathrm{d}, J_{\mathrm{C}-\mathrm{P}}=5.8 \mathrm{~Hz}\right), 168.35,133.85,132.03,123.14,62.56$ $\left(\mathrm{d}, J_{\mathrm{C}-\mathrm{P}}=6.5 \mathrm{~Hz}\right), 43.14,42.37\left(\mathrm{~d}, J_{\mathrm{C}-\mathrm{P}}=126.1 \mathrm{~Hz}\right), 37.43$, $27.69,20.42,16.23\left(\mathrm{~d}, J_{\mathrm{C}-\mathrm{P}}=5.8 \mathrm{~Hz}\right)$; HRMS (ESI-TOF) calcd for $\mathrm{C}_{18} \mathrm{H}_{24} \mathrm{NO}_{6} \mathrm{P}[\mathrm{M}+\mathrm{H}]^{+}$382.1416, found 382.1417 .

1-对甲苯磺酰胺基-3-氧代丁基膦酸二乙酯(2s): 淡 黄色油状物. ${ }^{1} \mathrm{H}$ NMR (400 MHz, $\left.\mathrm{CDCl}_{3}\right) \delta: 7.72(\mathrm{~d}, J=$ $8.0 \mathrm{~Hz}, 2 \mathrm{H}), 7.31(\mathrm{~d}, J=8.4 \mathrm{~Hz}, 2 \mathrm{H}), 5.42(\mathrm{t}, J=6.4 \mathrm{~Hz}$, $1 \mathrm{H}), 4.16 \sim 4.08(\mathrm{~m}, 4 \mathrm{H}), 3.15$ (q, $J=6.0 \mathrm{~Hz}, 2 \mathrm{H}), 3.05$ (d, $\left.J_{\mathrm{H}-\mathrm{P}}=22.8 \mathrm{~Hz}, 2 \mathrm{H}\right), 2.88(\mathrm{t}, J=5.8 \mathrm{~Hz}, 2 \mathrm{H}), 2.43$ (s, $3 \mathrm{H}), 1.33$ (t, $J=7.0 \mathrm{~Hz}, 6 \mathrm{H}) ;{ }^{13} \mathrm{C} \mathrm{NMR}\left(100 \mathrm{MHz}, \mathrm{CDCl}_{3}\right)$ $\delta: 201.25\left(\mathrm{~d}, J_{\mathrm{C}-\mathrm{P}}=6.6 \mathrm{~Hz}\right), 143.36,136.85,129.71$, $126.98,62.82\left(\mathrm{~d}, J_{\mathrm{C}-\mathrm{P}}=6.6 \mathrm{~Hz}\right), 43.53,42.54\left(\mathrm{~d}, J_{\mathrm{C}-\mathrm{P}}=\right.$ $126.1 \mathrm{~Hz}), 37.91,21.45,16.23\left(\mathrm{~d}, J_{\mathrm{C}-\mathrm{P}}=5.8 \mathrm{~Hz}\right) ; \mathrm{HRMS}$ (ESI-TOF) calcd for $\mathrm{C}_{15} \mathrm{H}_{24} \mathrm{NO}_{6} \mathrm{PS}[\mathrm{M}+\mathrm{H}]^{+}$377.1413, found 377.1416.

2-氧代-环已烯基乙基膦酸二乙酯 $(2 \mathrm{t})^{[26]}$ : 淡黄色油 状物. ${ }^{1} \mathrm{H}$ NMR $\left(400 \mathrm{MHz}, \mathrm{CDCl}_{3}\right) \delta: 7.01 \sim 6.96(\mathrm{~m}, 1 \mathrm{H})$, $4.73 \sim 4.61(\mathrm{~m}, 2 \mathrm{H}), 3.25\left(\mathrm{~d}, J_{\mathrm{H}-\mathrm{P}}=22.7 \mathrm{~Hz}, 2 \mathrm{H}\right), 2.28 \sim$ $2.22(\mathrm{~m}, 2 \mathrm{H}), 2.21 \sim 2.15(\mathrm{~m}, 2 \mathrm{H}), 1.63 \sim 1.53(\mathrm{~m}, 4 \mathrm{H}$, overlap), 1.28 (d, $J=6.2 \mathrm{~Hz}, 6 \mathrm{H}$, overlap), 1.27 (d, $J=6.2$ $\mathrm{Hz}, 6 \mathrm{H}$, overlap); ${ }^{13} \mathrm{C}$ NMR (100 MHz, $\left.\mathrm{CDCl}_{3}\right) \delta: 192.8$ (d,
$\left.J_{\mathrm{C}-\mathrm{P}}=6.1 \mathrm{~Hz}\right), 143.4,139.4,71.3\left(\mathrm{~d}, J_{\mathrm{C}-\mathrm{P}}=6.7 \mathrm{~Hz}\right), 38.5$ $\left(\mathrm{d}, J_{\mathrm{C}-\mathrm{P}}=131.3 \mathrm{~Hz}\right), 26.3,24.2\left(\mathrm{~d}, J_{\mathrm{C}-\mathrm{P}}=3.9 \mathrm{~Hz}\right), 23.9(\mathrm{~d}$, $J_{\mathrm{C}-\mathrm{P}}=5.2 \mathrm{~Hz}$ ), 23.3, 21.8, 21.6; HRMS (ESI-TOF) calcd for $\mathrm{C}_{12} \mathrm{H}_{21} \mathrm{O}_{4} \mathrm{P}[\mathrm{M}+\mathrm{H}]^{+}$260.1175, found 260.1174 .

2-环丙基-2-氧代乙基膦酸二乙酯(2u) ${ }^{[33]}$ : 淡黄色油 状物. ${ }^{1} \mathrm{H}$ NMR $\left(400 \mathrm{MHz}, \mathrm{CDCl}_{3}\right) \delta: 4.12 \sim 4.05(\mathrm{~m}, 4 \mathrm{H})$, $3.12\left(\mathrm{~d}, J_{\mathrm{H}-\mathrm{P}}=22.4 \mathrm{~Hz}, 2 \mathrm{H}\right), 2.14 \sim 2.07(\mathrm{~m}, 1 \mathrm{H}), 1.25(\mathrm{t}$, $J=7.2 \mathrm{~Hz}, 6 \mathrm{H}), 1.06 \sim 1.02(\mathrm{~m}, 2 \mathrm{H}), 0.93 \sim 0.88(\mathrm{~m}, 2 \mathrm{H})$; ${ }^{13} \mathrm{C}$ NMR $\left(100 \mathrm{MHz}, \mathrm{CDCl}_{3}\right) \delta: 201.97\left(\mathrm{~d}, J_{\mathrm{C}-\mathrm{P}}=5.9 \mathrm{~Hz}\right)$, $62.35\left(\mathrm{~d}, J_{\mathrm{C}-\mathrm{P}}=5.8 \mathrm{~Hz}\right), 43.08\left(\mathrm{~d}, J_{\mathrm{C}-\mathrm{P}}=126.9 \mathrm{~Hz}\right), 21.53$ $\left(\mathrm{d}, J_{\mathrm{C}-\mathrm{P}}=5.8 \mathrm{~Hz}\right), 16.15\left(\mathrm{~d}, J_{\mathrm{C}-\mathrm{P}}=6.5 \mathrm{~Hz}\right), 11.93$; HRMS (ESI-TOF) calcd for $\mathrm{C}_{9} \mathrm{H}_{17} \mathrm{O}_{4} \mathrm{P}[\mathrm{M}+\mathrm{H}]^{+} 220.0865$, found 220.0856 .

(2-苯乙基-2-氧代)膦酸二甲酯 $(2 \mathbf{v})^{[26]}$ : 淡黄色油状 物. ${ }^{1} \mathrm{H}$ NMR $\left(400 \mathrm{MHz}, \mathrm{CDCl}_{3}\right) \delta: 8.01(\mathrm{~d}, J=8.6 \mathrm{~Hz}$, 2H), $7.58(\mathrm{t}, J=7.4 \mathrm{~Hz}, 1 \mathrm{H}), 7.47(\mathrm{t}, J=7.4 \mathrm{~Hz}, 2 \mathrm{H}), 3.76$ (d, $J=11.2 \mathrm{~Hz}, 6 \mathrm{H}), 3.65(\mathrm{~d}, J=22.6 \mathrm{~Hz}, 2 \mathrm{H}) ;{ }^{13} \mathrm{C} \mathrm{NMR}$ $\left(100 \mathrm{MHz}, \mathrm{CDCl}_{3}\right) \delta: 191.8\left(\mathrm{~d}, J_{\mathrm{C}-\mathrm{P}}=6.6 \mathrm{~Hz}\right), 136.4(\mathrm{~d}$, $\left.J_{\mathrm{C}-\mathrm{P}}=2.3 \mathrm{~Hz}\right), 133.9,129.1,128.8,53.2\left(\mathrm{~d}, J_{\mathrm{C}-\mathrm{P}}=6.5 \mathrm{~Hz}\right)$, $37.6\left(\mathrm{~d}, J_{\mathrm{C}-\mathrm{P}}=131.2 \mathrm{~Hz}\right.$ ); HRMS (ESI-TOF) calcd for $\mathrm{C}_{10} \mathrm{H}_{14} \mathrm{O}_{4} \mathrm{P}[\mathrm{M}+\mathrm{H}]^{+}$229.0630, found 229.0635.

(2-苯乙基-2-氧代)膦酸二异丙酯 $(2 \mathbf{w})^{[20]}$ : 淡黄色油 状物. ${ }^{1} \mathrm{H}$ NMR $\left(400 \mathrm{MHz}, \mathrm{CDCl}_{3}\right) \delta: 8.00(\mathrm{~d}, J=7.7 \mathrm{~Hz}$, $2 \mathrm{H}), 7.58(\mathrm{t}, J=7.3 \mathrm{~Hz}, 1 \mathrm{H}), 7.48(\mathrm{t}, J=7.7 \mathrm{~Hz}, 2 \mathrm{H})$, $4.74 \sim 4.69(\mathrm{~m}, 2 \mathrm{H}), 3.58(\mathrm{~d}, J=23.1 \mathrm{~Hz}, 2 \mathrm{H}), 1.27$ (t, $J=$ $5.1 \mathrm{~Hz}, 12 \mathrm{H}) ;{ }^{13} \mathrm{C}$ NMR $\left(100 \mathrm{MHz}, \mathrm{CDCl}_{3}\right) \delta: 192.1$, $136.8,133.4,129.2,128.5,71.6\left(\mathrm{~d}, J_{\mathrm{C}-\mathrm{P}}=6.8 \mathrm{~Hz}\right), 39.8(\mathrm{~d}$, $J_{\mathrm{C}-\mathrm{P}}=130.0 \mathrm{~Hz}$ ), 23.8, 23.6; HRMS (ESI-TOF) calcd for $\mathrm{C}_{14} \mathrm{H}_{21} \mathrm{O}_{4} \mathrm{P}[\mathrm{M}+\mathrm{H}]^{+}$284.1174, found 284.1170.

(2-苯乙基-2-氧代)膦酸二正丁酯 $(\mathbf{2 x})^{[20]}$ : 淡黄色油 状物. ${ }^{1} \mathrm{H}$ NMR (400 MHz, $\left.\mathrm{CDCl}_{3}\right) \delta: 8.02(\mathrm{~d}, J=7.7 \mathrm{~Hz}$, $2 \mathrm{H}), 7.59$ (t, $J=7.5 \mathrm{~Hz}, 1 \mathrm{H}), 7.48(\mathrm{t}, J=7.7 \mathrm{~Hz}, 2 \mathrm{H})$, $4.11 \sim 4.03(\mathrm{~m}, 4 \mathrm{H}), 3.62(\mathrm{~d}, J=22.7 \mathrm{~Hz}, 2 \mathrm{H}), 1.63 \sim 1.57$ (m, 4H), $1.35 \sim 1.28(\mathrm{~m}, 4 \mathrm{H}), 0.89(\mathrm{t}, J=7.5 \mathrm{~Hz}, 6 \mathrm{H}) ;{ }^{13} \mathrm{C}$ NMR $\left(100 \mathrm{MHz}, \mathrm{CDCl}_{3}\right) \delta: 191.8,136.7,133.5,129.1$, $128.7,66.2\left(\mathrm{~d}, J_{\mathrm{C}-\mathrm{P}}=6.8 \mathrm{~Hz}\right), 38.5\left(\mathrm{~d}, J_{\mathrm{C}-\mathrm{P}}=128.9 \mathrm{~Hz}\right)$, $32.2\left(\mathrm{~d}, J_{\mathrm{C}-\mathrm{P}}=5.7 \mathrm{~Hz}\right), 18.5,13.6$; HRMS (ESI-TOF) calcd for $\mathrm{C}_{16} \mathrm{H}_{25} \mathrm{O}_{4} \mathrm{P}[\mathrm{M}+\mathrm{H}]^{+}$312.1491, found 312.1487.

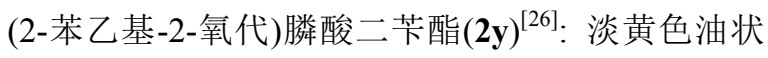
物. ${ }^{1} \mathrm{H}$ NMR (400 MHz, $\left.\mathrm{CDCl}_{3}\right) \delta: 7.86 \sim 7.83(\mathrm{~m}, 2 \mathrm{H})$, $7.46 \sim 7.42(\mathrm{~m}, 1 \mathrm{H}), 7.34 \sim 7.31(\mathrm{~m}, 2 \mathrm{H}), 7.23 \sim 7.17(\mathrm{~m}$, $10 \mathrm{H}), 5.01 \sim 4.90(\mathrm{~m}, 4 \mathrm{H}), 3.54\left(\mathrm{~d}, J_{\mathrm{H}-\mathrm{P}}=22.8 \mathrm{~Hz}, 2 \mathrm{H}\right)$; ${ }^{13} \mathrm{C}$ NMR $\left(100 \mathrm{MHz}, \mathrm{CDCl}_{3}\right) \delta: 191.25\left(\mathrm{~d}, J_{\mathrm{C}-\mathrm{P}}=6.6 \mathrm{~Hz}\right)$, $136.31\left(\mathrm{~d}, J_{\mathrm{C}-\mathrm{P}}=2.2 \mathrm{~Hz}\right), 135.75\left(\mathrm{~d}, J_{\mathrm{C}-\mathrm{P}}=5.8 \mathrm{~Hz}\right), 133.55$, $128.88,128.48,128.42,128.34,127.87,67.91\left(\mathrm{~d}, J_{\mathrm{C}-\mathrm{P}}=6.6\right.$ 
$\mathrm{Hz}), 38.50\left(\mathrm{~d}, J_{\mathrm{C}-\mathrm{P}}=130.5 \mathrm{~Hz}\right)$; HRMS (ESI-TOF) calcd for $\mathrm{C}_{22} \mathrm{H}_{21} \mathrm{O}_{4} \mathrm{P}[\mathrm{M}+\mathrm{H}]^{+}$380.1178, found 380.1174 .

\section{References}

[1] Lentsch, L. M.; Wiemer, D. F. J. Org. Chem. 1999, 64, 5205.

[2] Liu, Y.-L.; Liang, Y.; Pi, S.-F.; Li, J.-H. J. Org. Chem. 2009, 74, 5691.

[3] Kondoh, A.; Yorimitsu, H.; Oshima, K. Chem. Asian J. 2010, 5, 398

[4] Atmani, A.; Memmou, F.; Bouillon, J.-P. Cr. Chim. 2009, 12, 963.

[5] Whitten, J. P.; Cube, R. V.; Baron, B. M.; McDonald, I. A. Bioorg. Med. Chem. Lett. 1993, 3, 19.

[6] Liu, P.; Liu, A.; Yan, F.; Wolfe, M. D.; Lipscomb, J. D.; Liu, H.-W. Biochemistry 2003, 42, 11577.

[7] Li, X.; Bhandari, A.; Holmes, C. P.; Szardenings, A. K. Bioorg. Med. Chem. Lett. 2004, 14, 4301.

[8] Balg, C.; Blais, S. P.; Bernier, S.; Huot, J. L.; Couture, M.; Lapointe, J.; Chênevert, R. Biorg. Med. Chem. 2007, 15, 295.

[9] Perumal, S. K.; Adediran, S. A.; Pratt, R. F. Biorg. Med. Chem. 2008, 16, 6987.

[10] Maryanoff, B. E.; Reitz, A. B. Chem. Rev. 1989, 89, 863.

[11] Pronin, S. V.; Martinez, A.; Kuznedelov, K.; Severinov, K.; Shuman, H. A.; Kozmin, S. A. J. Am. Chem. Soc. 2011, 133, 72.

[12] Yang, W.; Yu, Y.; Zhang, T.; Hansmann, M. M.; Pflästerer, D.; Hashmi, A. S. K. Adv. Synth. Catal. 2013, 355, 2037.

[13] Kitamura, M.; Tokunaga, M.; Noyori, R. J. Am. Chem. Soc. 1995, 117, 2931.

[14] Chávez, M. Á.; Vargas, S.; Suárez, A.; Álvarez, E.; Pizzano, A. Adv. Synth. Catal. 2011, 353, 2775.

[15] Ryglowski, A.; Kafarski, P. Tetrahedron 1996, 52, 10685.
[16] McCabe, D. J.; Duesler, E. N.; Paine, R. T. Inorg. Chem. 1985, 24, 4626.

[17] Koprowski, M.; Szymańska, D.; Bodzioch, A.; Marciniak, B.; Różycka-Sokołowska, E.; Bałczewski, P. Tetrahedron 9, 65, 4017.

[18] Maloney, K. M.; Chung, J. Y. L. J. Org. Chem. 2009, 74, 7574.

[19] Demmer, C. S.; Krogsgaard-Larsen, N.; Bunch, L. Chem. Rev. 2011, 111, 7981.

[20] Wei, W.; Ji, J.-X. Angew. Chem., Int. Ed. 2011, 50, 9097.

[21] Beller, M.; Seayad, J.; Tillack, A.; Jiao, H. Angew. Chem., Int. Ed. 2004, 43, 3368.

[22] Hintermann, L.; Labonne, A. Synthesis 2007, 1121.

[23] Zeng, X. Chem. Rev. 2013, 113, 6864.

[24] Xie, L. Y.; Yuan R.; Wang R. J.; Peng, Z. H.; Xiang, J. N.; He, W. M. Eur. J. Org. Chem. 2014, 13, 2668.

[25] Sturtz, C. C. G.; Normant, H. Bull. Soc. Chim. Fr. 1966, 1707.

[26] Li, X.; Hu, G.; Luo, P.; Tang, G.; Gao, Y.; Xu, P.; Zhao, Y. Adv. Synth. Catal. 2012, 354, 2427.

[27] Liu, K. J.; Liu, H. W.; Wang, W. G.; Ou, L. J.; Wang, J. J.; Hu, B. N. Chin. J. Org. Chem. 2014, 34, 2007 (in Chinese). (刘开建, 刘宏伟, 王文革, 欧丽娟, 王津津, 胡波年, 有机化学, 2014, 34, 2007.)

[28] Liu, K. J. Chem. J. Chin. Univ. 2014, 35, 1236 (in Chinese). (刘开建, 高等学校化学学报, 2014, 35, 1236.)

[29] Leyva, A.; Corma, A. J. Org. Chem. 2009, 74, 2067.

[30] Thuong, M. B. T.; Mann, A.; Wagner, A. Chem. Commun. 2012, 48, 434.

[31] Luke, G. P.; Seekamp, C. K.; Wang, Z.-Q.; Chenard, B. L. J. Org. Chem. 2008, 73, 6397.

[32] Moorhoff, C. M. Synth. Commun. 2003, 33, 2069.

Orsini, F.; Teodoro, E. D.; Ferrari, M. Synthesis 2002, 1683.

(Li, L.; Lu, Z.) 